\title{
Nouvelles règles d'évaluation des produits phytopharmaceutiques : conséquences pour les oléagineux
}

\author{
Fabien LAGARDE \\ CETIOM, Direction Technique, \\ Centre de Grignon, avenue Lucien Bretignière, \\ 78850 Thiverval Grignon \\ <lagarde@cetiom.fr>
}

\begin{abstract}
At the beginning of 2009, Parliament has adopted two directives to reduce utilizations of pesticides in Europe. Some Cut off criteria will be used after 2011 for the approval of active substances. This decision will affect the possibilities of chemical protection for the oilseed. A Pesticides Safety Directorate study shows that several herbicides, fungicides and insecticides actually used will be suspended, but without major impact for the crop protection, especially if expected innovations will be available next years.
\end{abstract}

Key words: pesticides, Europe, oilseed

- la mise en place d'actions de formation auprès des acteurs de la protection des plantes et de sensibilisation auprès du grand public ;

- la mise en place d'un système d'inspection des matériels d'application et l'interdiction des applications aériennes avec mesures dérogatoires à la marge ;

- la mise en place de mesure de protection des milieux aquatiques et milieux sensibles ainsi que les espaces publics ou fréquentés par des populations sensibles;

- la mise en place de mesures visant à limiter I'usage des pesticides avec l'objectif de parvenir à la protection intégrée des cultures à partir de 2014;

- des mesures spécifiques visant à sécuriser la manipulation et stockage des produits ;

- et enfin, la mise en place d'indicateurs harmonisés de risque et des statistiques permettant de suivre l'efficacité des mesures prises aux niveaux national et européen.

La future Directive-cadre remplaçant l'actuelle CE 91/414 a sensiblement évolué notamment avec l'adoption de deux principes nouveaux pour évaluer les produits de protection des plantes:

- l'évaluation des produits de protection des plantes portera essentiellement sur la notion de danger « cut-off criteria » alors que jusqu'à présent elle s'appuyait sur la notion de risque résultant de l'analyse conjointe du danger associé au niveau d'exposition ;

- le principe de substitution est introduit. Il consiste à substituer les molécules les plus dangereuses par des molécules à meilleur profil lorsque c'est possible.

Les critères d'exclusion qui ont été retenus quant à eux, concernent :
- la protection de la santé humaine, avec l'exclusion des produits classés Cancérigène, Mutagène, ou effet sur la Reproduction de catégorie 1 et 2 selon la Directive 67/548/EEC, (avec exception possible, si l'exposition est négligeable) ou si le produit est considéré comme perturbateur endocrinien. La méthode qui sera utilisée pour évaluer ce critère reste encore à établir

- la protection de l'environnement, avec les critères d'exclusion suivants: OP (organic pollutant), PBT (persistent, bioaccumulating, and toxic), VPVB (very persistent, very bioaccumulating).

Cette nouvelle Directive doit entrer en vigueur à partir du premier trimestre 2011 pour les nouvelles demandes d'inscription de substances actives déposées par les sociétés phytosanitaires. Les molécules actuelles, qui ont été évaluées dans l'actuelle 91/414, seront pour les premières d'entre elles soumises à réévaluation dans cette nouvelle procédure au plus tôt à partir de 2015.

\section{Impact des « cut off criteria " : encore des incertitudes}

Une analyse d'impact de ces mesures a été réalisée par le PSD (Pesticides Safety Directorate) en mai 2008 en Angleterre. En raison de l'incertitude concernant le critère portant sur l'effet " perturbateur endocrinien », dont l'évaluation précise n'est pas encore fixée, les résultats sont à considérer avec précaution et sont donnés à titre indicatif sachant que l'impact lié à ce critère peut être assez différent selon le niveau retenu. 
D’une manière générale, pour les oléagineux, les nouvelles mesures adoptées pour l'évaluation des produits phytosanitaires semblent moins préoccupantes que pour d'autres filières grandes cultures, comme les céréales par exemple.

Pour les substances insecticides, qui constituent un secteur de la protection des cultures stratégique pour les oléagineux, l'impact de l'évolution de la réglementation permet d'envisager le maintien des pyréthrinoïdes actuellement disponibles qui sont à la base de la protection. Cependant, la bifenthrine, qui est l'une des seules matières actives efficaces avec le taufluvalinate pour lutter contre le méligèthe est concernée pour les critères PBT (persistent, bioaccumulating and toxic) et VPvB (very Persistent, very Bioaccumulating). Par ailleurs, la deltaméthrine pourrait être concernée par le critère perturbateur endocrinien. Par contre, un plus grand nombre de matières actives sont visées par la substitution, comme par exemple et non des moindres, le tau fluvalinate (efficace sur méligèthes), la lambda cyhalothrine, la deltamethrine ou le pyrimicarbe (seule solution encore efficace sur pucerons verts et pucerons cendrés). II est important de noter que la protection insecticide repose presque exclusivement sur la famille des pyréthrinoïdes. Un nombre encore plus limité de matières actives accentue donc nettement le risque d'apparition de résistances en culture.

Pour les fongicides, ceux qui sont concernés par leur classement R2 (effet sur la reproduction) sont d'ores et déjà en cours de retrait ou retirés pour les usages oléagineux comme par exemple la carbendazime, la procymidone ou le flusilazole. Si l'on prend en compte le critère perturbateur endocrinien pour lequel une incertitude existe, alors, il semble que tous les fongicides de la famille des triazoles (ayant un effet fongicide et régulateur) soient concernés, ainsi que le thirame qui est l'unique traitement de semences colza utilisé. Par contre, nous ne disposons pas d'information sur les nouveaux fongicides qui sont apparus comme le Boscalid ou le prothioconalzole. La famille des strobilurines est quant à elle préservée. Donc, vis-à-vis des principales maladies qui sévissent sur oléagineux en France, des solutions sont préservées, même si parfois le nombre de familles de matières actives disponibles est insuffisant pour gérer durablement les risques d'apparition de résistances.

Pour les herbicides, le linuron classé R2, à la base du désherbage dans les milieux à faible potentiel en tournesol, ne satisfait pas aux nouveaux critères. La pendiméthaline classée PBT également utilisée sur tournesol en programme ne serait plus disponible. La perte de cette matière active serait très préjudiciable en l'absence d'innovation, car avec la disparition récente de la trifluraline à la base des programmes de désherbage du tournesol, celle-ci constitue une alternative intéressante en particulier contre graminées.

Enfin, le piclorame qui pourrait être perturbateur endocrinien serait également concerné. Cette matière active est utilisée sur colza dans les secteurs à forte infestation de géraniums et gaillets comme solution de rattrapage de post-levée.

Ainsi, l'évolution envisagée de la Directivecadre 91/414 ne générera pas d'impasse technique. Par contre, en l'absence d'innovation, le désherbage des oléagineux qui ne dispose $\mathrm{d}^{\prime}$ ores et déjà que de peu de matières actives sera fragilisé tout comme la lutte contre les maladies, si l'on prend en compte le risque d'apparition de résistance. La lutte contre les insectes, qui représentent une menace très importante pour le colza, est actuellement confrontée à des phénomènes de résistance (pucerons, méligèthes) avec les pyréthrinoïdes de synthèse. Elle devra donc pouvoir dans un proche avenir élargir sensiblement la gamme des matières actives disponibles, pour garder des solutions efficaces. II semble que les nouveaux critères adoptés ne constituent pas un frein, puisque des produits de la famille des organophosphorés et des néoniotinoïdes actuellement disponibles en Europe - mais pas en France - seraient préservés. 